\title{
Association Between Statin Use and Exacerbation of Chronic Obstructive Pulmonary Disease Among Patients Receiving Corticosteroids
}

This article was published in the following Dove Press journal: International Journal of Chronic Obstructive Pulmonary Disease

\author{
Yi-Jen Huang $\mathbb{D}^{1,2}$ \\ Senyeong $\mathrm{KaO}^{1,3}$ \\ Li-Ting Kao (iD ${ }^{1,3-5}$ \\ Chih-Feng Chian ${ }^{6}$ \\ Chi-Hsiang Chung $\mathbb{D D}^{3,7}$ \\ Wu-Chien Chien (iD ${ }^{1,3,7 *}$ \\ Kwua-Yun Wang ${ }^{1,8,9 *}$ \\ 'Graduate Institute of Life Sciences, National \\ Defense Medical Center, Taipei, Taiwan; \\ ${ }^{2}$ Department of Nursing, University of Kang \\ Ning, Taipei, Taiwan; ${ }^{3}$ School of Public Health, \\ National Defense Medical Center, Taipei, \\ Taiwan; ${ }^{4}$ Department of Pharmacy Practice, \\ Tri-Service General Hospital, Taipei, Taiwan; \\ ${ }^{5}$ School of Pharmacy, National Defense \\ Medical Center, Taipei, Taiwan; ${ }^{6}$ Division of \\ Pulmonary and Critical Care Medicine, \\ Department of Internal Medicine, Tri-Service \\ General Hospital, National Defense Medical \\ Center, Taipei, Taiwan; ${ }^{7}$ Department of \\ Medical Research, Tri-Service General \\ Hospital, National Defense Medical Center, \\ Taipei, Taiwan; ${ }^{8} \mathrm{~S} c h o o l$ of Nursing, National \\ Defense Medical Center, Taipei, Taiwan; \\ ${ }^{9}$ Department of Nursing, Taipei Veterans \\ General Hospital, Taipei, Taiwan
}

*These authors contributed equally to this work

Correspondence: Wu-Chien Chien Department of Medical Research, TriService General Hospital, National Defense Medical Center, 7I I5R, No. 325, Section 2, Cheng-Kung Road, Neihu District, Taipei

City, I 1490, Taiwan, Republic of China

Tel +886-2-879233I I, Ext. 19189

Fax +886-2-87927235

Email chienwu@ndmctsgh.edu.tw

Kwua-Yun Wang

Graduate Institute of Life Sciences, National Defense Medical Center, 93I4R, No. I6I,

Section 6, Min-Chuan East Road, Neihu

District, Taipei City, I 1490, Taiwan

Tel +886-2-87923100 ext. 18766

Email kywang7@vghtpe.gov.tw
Purpose: The role of statins as anti-inflammatory drugs in chronic obstructive pulmonary disease (COPD) is controversial. This study aimed to determine the efficacy of statins used with or without corticosteroids in COPD patients.

Patients and Methods: This was a retrospective cohort study and used the two million outpatients and inpatients in Taiwan's Longitudinal Health Insurance Database covering 2000 to 2015 . A total of 92,460 patients were identified in this study. We divided COPD patients into four groups by auditing each patient's medication (statins used or not; corticosteroids used or not) and used Cox regression to analyze and compare the effects of statins in COPD patients with or without corticosteroids.

Results: In terms of all COPD patients, our findings were consistent with previous studies showing that statins decreased COPD-related hospitalization and mortality rates. However, the beneficial effects were only observed in younger patients or those not taking corticosteroids in further analysis. Statins significantly decreased hospitalization and mortality rates in the non-corticosteroids groups. The hazard ratios increased with age and were not statistically significant for patients $>70$ years old. Statins did not significantly decrease ED visits, hospitalization, and mortality in corticosteroids groups.

Conclusion: Statins decreased hospitalization and mortality rates in COPD patients not taking corticosteroids but were not efficacious in patients on corticosteroids therapy. Furthermore, the beneficial effects of statins gradually decreased with patient age. Based on the findings, statins used in COPD patients may need to consider the patient age and corticosteroids used or not.

Keywords: COPD, exacerbation, statins, corticosteroids, mortality

\section{Introduction}

According to the World Health Organization, chronic obstructive pulmonary disease (COPD) caused 3.0 million lives in 2016 and is the third leading cause of death globally. According to the Global Initiative for Chronic Obstructive Lung Disease (GOLD), COPD is a lung disease that causes long-term inflammation, an inexorable decline in respiratory function, and eventually death because current treatments cannot prevent disease progression. ${ }^{1}$ The standard medications used long-term to manage COPD and prevent exacerbation are bronchodilators, corticosteroids, and phosphodiesterase-4 inhibitors. Although the GOLD has modified its guidelines every year, prevention of disease progression has still not been achieved. 
Statins are 3-hydroxy-3-methylglutaryl coenzyme A reductase inhibitors used as hypolipidemic agents. Statins were also reported to provide anti-inflammatory effects, preventing the recurrence of multiple diseases, including COPD. ${ }^{2-9}$ Most studies about statins have shown beneficial effects in COPD but could not determine which patient groups can best benefit from statin therapy. ${ }^{10,11}$ Other studies have reported that many situations can modify the effect of statins, such as COPD severity, sex, current medication, age and comorbidities. ${ }^{9}$ Moreover, a prospective trial concluded that the efficacy of statins was not effective in moderate-to-severe COPD. ${ }^{12}$ Therefore, given these different results, it is possible that other factors have contributed to the differences in the results for the efficacy of statins in the treatment of COPD.

Corticosteroids have long been used as major maintenance drugs for more severe COPD (GOLD staging system, groups $\mathrm{C}$ and $\mathrm{D}$, including less pulmonary function left, more exacerbations history or hospitalization requirement, or worse eosinophilia) to reduce inflammation. ${ }^{1}$ Corticosteroids possess a powerful immune-modulatory effect and are a clinical mainstay for the treatment of numerous inflammatory diseases, including autoimmune diseases. Considering the potential pharmacological mechanism, statins it is possible that statins should not be recommended as an additional anti-inflammatory drug in the treatment of COPD patients who already take corticosteroids. The aim of this study was to determine if there are differences in the efficacy of statins in COPD patient groups also receiving or not receiving corticosteroids therapy.

\section{Patients and Methods Study Design and Data Source}

This was a retrospective study in a cohort extracted from the records of the two million outpatients and inpatients in Taiwan's Longitudinal Health Insurance Database (LHID) covering 2000 to 2015. This LHID two million-person data sample was randomly selected from the Taiwan National Health Insurance Research Database (NHIRD), which includes $>99 \%$ of the 23 million inhabitants of Taiwan and contains all their medical records. For privacy protection, all data were scrambled and translated so that individuals could not be identified at any level of use. The data source was provided by the Health and Welfare Data Science Center, Ministry of Health and Welfare. This study was approved and exempted from review by Institutional Review Board I of the Tri-Service General Hospital, National Defense Medical Center (TSGHIRB No.: B-108-12) because of anonymized databases used only.

\section{Cohort Identification}

Initially, we identified COPD patients who had received medical codes of ICD-9-CM 491-492, 496 three times or more, and were $>40$ years old (based on the most prevalent age for identifying the presence of COPD with differentiation from asthma). Patients who had received statins or had been diagnosed with COPD before 2000 were excluded. The remaining COPD patients were divided into four groups by auditing each patient's medication situation from the administrative claims database (with or without corticosteroids usage and concomitant use or nonuse of statins) (Figure 1). The four groups are as follows: 1) non-corticosteroids without statins, 2) non-corticosteroids with statins, 3) corticosteroids without statins and 4) corticosteroids with statins. Corticosteroids usage was defined as any form of ingestion and included local or systemic drugs. Statins were given only in oral form in this study. Statins or corticosteroids usage was defined as usage periods $>60$ days (Supplementary Table S1). The selection of the usage periods $>60$ days was based on the "refillable prescriptions for patients with chronic illnesses" policy. Patients should have to revisit medical institutions more than three times per year and maintain the prescribing as before. This design could ensure the stability of COPD patients' medication but also good compliance.

\section{Outcomes Definition}

COPD exacerbation was defined as the need for admission to the emergency department (ED) or for hospitalization related to COPD. Mortality was defined as all-cause mortality. Any outcomes occurring $<60$ days were excluded from the calculations. The detailed definitions of comorbidities and ICD-9-CM are presented in Supplementary Table S1. Given that COPD is a progressive illness, we rechecked the medication used by each patient for each outcome and excluded patients that did not meet our criteria for inclusion to avoid erroneous contributions to the outcome results from patients who may have not been taking corticosteroids at enrollment but were taking corticosteroids later because of disease progression (Supplementary Table S1). 


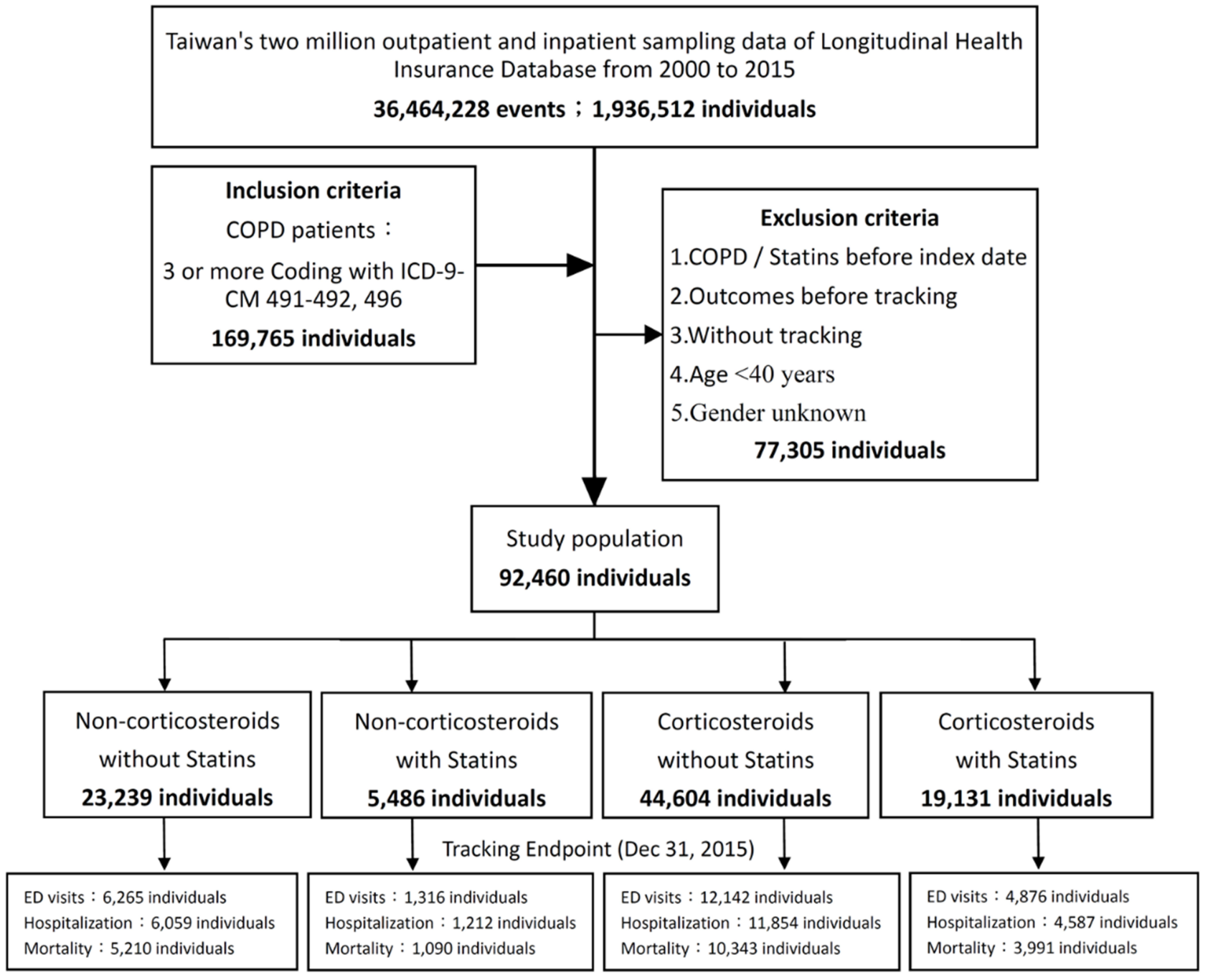

Figure I Flowchart of study cohort selection.

Notes: Inclusion criteria were codes of ICD-9-CM 49I-492, 496 three times or more, and were >40 years old; patients who had received statins or had been diagnosed with COPD before 2000 were excluded; the remaining patients were divided into four groups (with or without corticosteroids usage and concomitant use or non-use of statins) to analyze.

Abbreviations: COPD, chronic obstructive pulmonary disease; ED, emergency department.

\section{Statistical Analysis}

Data were analyzed using SPSS (version 22) statistical software (SPSS Software, IBM Corp., Armonk, NY, USA). Comparisons of covariates were performed by using the chi-square and Fisher exact tests for categorical variables and one-way ANOVA with Scheffe's post hoc test for continuous variables. Cox regression models were used to estimate the hazard ratio after proper adjustments for various factors and comorbidities. Further analysis with stratification by sex and age was performed. Kaplan-Meier analysis with the Log-rank test was performed to determine the cumulative incidence and mortality among the different cohorts. Two-sided $p$ values $<0.05$ were taken to be indicative of statistical significance. To check the reproducibility of the result, we performed the analysis again after further propensity score matching.

\section{Results}

\section{Study Description and Characteristics}

The flowchart of the study design is shown in Figure 1. A total of 92,460 patients were identified from the two million-person sample dataset of the LHID covering the period from 1, January, 2000 to 31, December, 2015. 
Approximately two-thirds of the COPD patients were taking corticosteroids when enrolled in the study $(n=63,735$, $68.93 \%$ ), and one-fourth of the COPD patients were receiving statins $(n=24,617,26.62 \%)$.

The baseline characteristics of the study patients are presented in Table 1. The mean age of the patients was 69.83 years, and $64.66 \%$ of the patients were males. The study population almost doubled in each decade since the 40-49-year-old group, and the peak was in the 70-79-year -old group (40.02\%), then decrease. More than half of the patients $(55.04 \%)$ were over 70 years old. In addition, the hazard ratios for visiting the ED [adjusted hazard ratios (aHR) 1.894, 95\% confidence interval (CI) 1.390-2.182, $p<0.001$ ], hospitalization (aHR 1.587, 95\% CI 1.304-$1.875, p<0.001$ ), and mortality (aHR 1.796, 95\% CI 1.493-1.930, $p<0.001)$ were higher in males than in females. The prognosis of COPD worsened with age. We examined the variables by Cox regression analysis, which revealed that most comorbidities aggravated the outcomes (Supplementary Table S2).

\section{Outcomes}

\section{COPD Exacerbation}

In all COPD patients, statins significantly decreased hospitalization (aHR 0.841, 95\% CI 0.748-0.902, $p<0.001$ ) and mortality rates (aHR $0.735,95 \%$ CI $0.535-0.886, p<0.001$ ) related to COPD (Table 2). However, when stratified by taking corticosteroids or not and compare to each other, the beneficial effects of statins were only observed in the noncorticosteroids groups. We found that statins did not significantly decrease the risk of COPD exacerbation for patients who were taking corticosteroids concomitantly, as shown by no significant decreases in ED visits (aHR 0.966, $95 \%$ CI $0.782-1.779, p=0.228$ ) or hospitalization rates (aHR 0.953, 95\% CI 0.751-1.498, $p=0.253$ ). However, statins did significantly decrease the risk of COPD hospitalization in the non-corticosteroids groups (aHR 0.779, 95\% CI $0.586-0.902, p<0.001)$ but did not significantly reduce ED visits (aHR 0.721, 95\% CI 0.511-1.006, $p=0.058$ ) (Table 2).

Moreover, Kaplan-Meier analysis with the Logrank test showed that the cumulative incidences of ED visits and hospitalization were lower in the non-corticosteroids groups receiving statins. By contrast, there were no significant differences in ED visits and hospitalization in the corticosteroids groups with or without concomitant use of statins (Supplementary Figures S1 and $\underline{\mathrm{S} 2}$ ). The results remain the same using propensity score matching 1:1 for analysis (Figure 2).

Furthermore, the effects of statins on COPD patients were similar between males and females in a stratified analysis. All of the hazard ratios gradually increased and differences became non-significance with age. Specifically, the hazard ratios for statins gradually increased from that of the youngest age group (40-49 age group), and differences became non-significant for patients $>70$ years old. In the corticosteroids with statins group $>70$ years old, the hazard ratios for use of statins became $>1$ and the effect was non-significant (Figure 3 and Supplementary Table $\underline{\mathrm{S}}$ ). It also revealed the same result in propensity score matching (Supplementary Table S4).

\section{All-Cause Mortality}

Overall, patients with COPD who received statins had a significantly decreased mortality rate (aHR 0.735, 95\% CI $0.535-0.886, p<0.001)$. However, we found that statins did not significantly decrease mortality in corticosteroids groups (aHR 0.932, 95\% CI 0.740-1.245, $p=$ 0.287 ), and there was a much greater decrease in the mortality rate at non-corticosteroids groups (aHR 0.668, $95 \%$ CI $0.389-0.895, p<0.001$ ) (Table 2). In terms of propensity score matching $1: 1$ for analysis, the result remains the same (aHR 0.944, 95\% CI 0.748-1.260, $p=$ 0.291 in corticosteroids groups and aHR $0.676,95 \% \mathrm{CI}$ $0.395-0.907, p<0.001$ in non-corticosteroids groups) (Figure 2). The effect of statins was the same for males and females, but the hazard ratios increased with age (Figure 3 and Supplementary Table S3). Kaplan-Meier analysis with the Logrank test showed the same result (Supplementary Figure S3).

\section{Discussion}

Our study found that statins significantly decreased hospitalization and mortality rates in the entire COPD population. These results were consistent with those of most previous studies. $^{2-9,13-15}$ However, the most important finding was that after stratifying that concomitant use or non-use of corticosteroids, statins were not found to be efficacious for decreasing COPD exacerbation (hospitalization rate or ED visits) and mortality rates in the corticosteroids groups. According to the COPD patient characteristics, which means statins were not efficacious in two-thirds of the entire COPD population (68.93\%).

To the best of our knowledge, only one previous study has mentioned that statins were not efficacious in COPD 
Table I Characteristics of Study in the Baseline

\begin{tabular}{|c|c|c|c|c|c|c|}
\hline \multirow[t]{2}{*}{ Cohort } & \multirow[t]{2}{*}{ Overall } & \multicolumn{2}{|c|}{ Non-Corticosteroids } & \multicolumn{2}{|c|}{ Corticosteroids } & \multirow[t]{2}{*}{$P$} \\
\hline & & Without Statins & With Statins & Without Statins & With Statins & \\
\hline Variable & $n=92,460(\%)$ & $n=23,239(\%)$ & $n=5486(\%)$ & $n=44,604(\%)$ & $n=19,131(\%)$ & \\
\hline \multicolumn{7}{|l|}{ Sex } \\
\hline Male & $60,075(64.66)$ & $15,134(64.81)$ & $3,361(61.27)$ & 28,901 (64.79) & $12,459(65.12)$ & $<0.001$ \\
\hline Female & $32,835(35.34)$ & $8335(35.19)$ & $2125(38.73)$ & $15,703(35.21)$ & $6672(34.88)$ & \\
\hline Age (years) & $69.83 \pm 10.78$ & $68.40 \pm 10.05$ & $69.03 \pm 10.20$ & $70.61 \pm 12.67$ & $70.10 \pm 11.55$ & $<0.001$ \\
\hline \multicolumn{7}{|c|}{ Age group (years) } \\
\hline $40-49$ & $5153(5.57)$ & $737(3.17)$ & $402(7.33)$ & $2801(6.28)$ & $1213(6.34)$ & $<0.001$ \\
\hline $50-59$ & $13,124(14.19)$ & $3311(14.25)$ & $678(12.36)$ & $6060(13.59)$ & $3075(16.07)$ & \\
\hline $60-69$ & $23,295(25.19)$ & $7169(30.85)$ & $1429(26.05)$ & 9972 (22.36) & $4725(24.70)$ & \\
\hline 70-79 & $37,001(40.02)$ & 8712 (37.49) & $2129(38.81)$ & 19,165 (42.97) & 6995 (36.56) & \\
\hline$\geqq 80$ & 13,887 (15.02) & $3310(14.24)$ & $848(15.46)$ & $6606(14.81)$ & $3123(16.32)$ & \\
\hline \multicolumn{7}{|c|}{ Catastrophic illness } \\
\hline Without & $81,112(87.73)$ & 21,123 (90.89) & $4964(90.48)$ & $38,628(86.60)$ & 16,397 (85.7I) & $<0.001$ \\
\hline With & 11,348 (12.27) & $2116(9.11)$ & $522(9.52)$ & $5976(13.40)$ & $2734(14.29)$ & \\
\hline \multicolumn{7}{|c|}{ Hypertension } \\
\hline Without & $70,464(76.21)$ & 18,589 (79.99) & $4,227(77.05)$ & 34,579 (77.52) & $13,069(68.31)$ & $<0.001$ \\
\hline With & $21,996(23.79)$ & $4650(20.01)$ & $1259(22.95)$ & $10,025(22.48)$ & $6062(31.69)$ & \\
\hline \multicolumn{7}{|c|}{ Myocardial infarction } \\
\hline Without & $89,042(96.30)$ & 22,707 (97.7I) & 5288 (96.39) & $42,729(95.80)$ & 18,318 (95.75) & $<0.001$ \\
\hline With & $3418(3.70)$ & $532(2.29)$ & $198(3.61)$ & $1875(4.20)$ & $813(4.25)$ & \\
\hline \multicolumn{7}{|c|}{ Congestive heart failure } \\
\hline Without & $86,850(93.93)$ & $22,279(95.87)$ & $5,185(94.5 I)$ & 41,607 (93.28) & 17,779 (92.93) & $<0.001$ \\
\hline With & $5610(6.07)$ & $960(4.13)$ & 301 (5.49) & $2997(6.72)$ & 1352 (7.07) & \\
\hline \multicolumn{7}{|c|}{ Rheumatologic disease } \\
\hline Without & $90,386(97.76)$ & $22,744(97.87)$ & $5,378(98.03)$ & 43,631 (97.82) & $18,633(97.40)$ & $<0.001$ \\
\hline With & $2074(2.24)$ & $495(2.13)$ & $108(1.97)$ & $973(2.18)$ & $498(2.60)$ & \\
\hline \multicolumn{7}{|l|}{ Dementia } \\
\hline Without & 89,205 (96.48) & 22,77I (97.99) & $534 I(97.36)$ & $42,852(96.07)$ & $|8,24|(95.35)$ & $<0.001$ \\
\hline With & $3255(3.52)$ & $468(2.01)$ & $145(2.64)$ & $1752(3.93)$ & $890(4.65)$ & \\
\hline \multicolumn{7}{|c|}{ Peripheral vascular disease } \\
\hline Without & $79,543(86.03)$ & 20,885 (89.87) & $4,886(89.06)$ & $37,570(84.23)$ & 16,202 (84.69) & $<0.001$ \\
\hline With & $12,917(13.97)$ & $2354(10.13)$ & $600(10.94)$ & $7034(15.77)$ & $2929(15.31)$ & \\
\hline \multicolumn{7}{|c|}{ Diabetes mellitus } \\
\hline Without & $76,600(82.85)$ & $21,382(92.01)$ & $4,662(84.98)$ & $36,491(81.81)$ & $14,065(73.52)$ & $<0.001$ \\
\hline With & $15,860(17.15)$ & I857 (7.99) & $824(15.02)$ & $8113(18.19)$ & $5066(26.48)$ & \\
\hline \multicolumn{7}{|c|}{ Diabetes with chronic complication } \\
\hline Without & $74,212(80.26)$ & $20,371(87.66)$ & $4487(81.79)$ & $35,358(79.27)$ & $13,996(73.16)$ & $<0.001$ \\
\hline With & $18,248(19.74)$ & $2868(12.34)$ & $999(18.21)$ & $9246(20.73)$ & $5135(26.84)$ & \\
\hline \multicolumn{7}{|c|}{ Pulmonary hypertension } \\
\hline Without & $80,684(87.26)$ & $21,283(91.58)$ & 4,816 (87.79) & $38,592(86.52)$ & $15,993(83.60)$ & $<0.001$ \\
\hline With & $11,776(12.74)$ & 1956 (8.42) & $670(12.21)$ & $6012(13.48)$ & $3138(16.40)$ & \\
\hline \multicolumn{7}{|c|}{ Cerebrovascular disease } \\
\hline Without & 79,121 (85.57) & 19,859 (85.46) & $47 \mid 7$ (85.98) & $38,516(86.35)$ & 16,029 (83.79) & $<0.001$ \\
\hline With & $13,339(14.43)$ & $3380(14.54)$ & 769 (14.02) & $6088(13.65)$ & $3102(16.21)$ & \\
\hline
\end{tabular}

(Continued) 
Table I (Continued).

\begin{tabular}{|c|c|c|c|c|c|c|}
\hline \multirow[t]{2}{*}{ Cohort } & \multirow[t]{2}{*}{ Overall } & \multicolumn{2}{|c|}{ Non-Corticosteroids } & \multicolumn{2}{|c|}{ Corticosteroids } & \multirow[t]{2}{*}{$P$} \\
\hline & & Without Statins & With Statins & Without Statins & With Statins & \\
\hline Variable & $n=92,460(\%)$ & $n=23,239(\%)$ & $n=5486(\%)$ & $n=44,604(\%)$ & $n=19,131(\%)$ & \\
\hline \multicolumn{7}{|c|}{ Hemiplegia/paraplegia } \\
\hline Without & $92,453(99.99)$ & $23,237(99.99)$ & $5486(100.00)$ & $44,603(100.00)$ & $19,127(99.98)$ & 0.018 \\
\hline With & $7(0.01)$ & $2(0.01)$ & $0(0.00)$ & I $(0.00)$ & $4(0.02)$ & \\
\hline \multicolumn{7}{|l|}{ Anemia } \\
\hline Without & 91,551 (99.02) & $23,092(99.37)$ & $5456(99.45)$ & $44,169(99.02)$ & 18,834 (98.45) & $<0.001$ \\
\hline With & $909(0.98)$ & $147(0.63)$ & $30(0.55)$ & $435(0.98)$ & $297(1.55)$ & \\
\hline \multicolumn{7}{|c|}{ Hyperlipidemia } \\
\hline Without & 84,656 (91.56) & 22,931 (98.67) & $4,356(79.40)$ & $43,179(96.81)$ & $14,190(74.17)$ & $<0.001$ \\
\hline With & $7804(8.44)$ & $308(1.33)$ & $1130(20.60)$ & $1425(3.19)$ & $494 \mathrm{I}(25.83)$ & \\
\hline \multicolumn{7}{|c|}{ Liver cirrhosis } \\
\hline Without & $78,609(85.02)$ & $20,038(86.23)$ & $4760(86.77)$ & 37,815 (84.78) & $15,996(83.61)$ & $<0.001$ \\
\hline With & $|3,85|(14.98)$ & $3201(13.77)$ & $726(13.23)$ & $6789(15.22)$ & $3135(16.39)$ & \\
\hline \multicolumn{7}{|c|}{ Moderate to server liver disease } \\
\hline Without & $78,224(84.60)$ & $19,945(85.83)$ & $4753(86.64)$ & $37,692(84.50)$ & I5,834 (82.77) & $<0.001$ \\
\hline With & $14,236(15.40)$ & $3294(14.17)$ & $733(13.36)$ & $6912(15.50)$ & $3297(17.23)$ & \\
\hline \multicolumn{7}{|c|}{ Renal disease } \\
\hline Without & $77,564(83.89)$ & 19,881 (85.55) & $4674(85.20)$ & $37,530(84.14)$ & $15,479(80.91)$ & $<0.001$ \\
\hline With & $14,896(16.11)$ & $3358(14.45)$ & $812(14.80)$ & $7074(15.86)$ & $3652(19.09)$ & \\
\hline \multicolumn{7}{|c|}{ Metastatic cancer } \\
\hline Without & $80,972(87.58)$ & $20,496(88.20)$ & $4898(89.28)$ & $39,481(88.51)$ & $16,097(84.14)$ & $<0.001$ \\
\hline With & $11,488(12.42)$ & $2743(11.80)$ & $588(10.72)$ & $5123(11.49)$ & $3034(15.86)$ & \\
\hline \multicolumn{7}{|c|}{ Malignancy (cancer), including leukemia and lymphoma } \\
\hline Without & $79,134(85.59)$ & $20,265(87.20)$ & $4797(87.44)$ & $38,613(86.57)$ & $15,459(80.81)$ & $<0.001$ \\
\hline With & $13,326(14.41)$ & $2974(12.80)$ & $689(12.56)$ & $5991(13.43)$ & $3672(19.19)$ & \\
\hline \multicolumn{7}{|c|}{ Peptic ulcer disease } \\
\hline Without & $89,067(96.33)$ & $22,560(97.08)$ & $5310(96.79)$ & $42,979(96.36)$ & $18,218(95.23)$ & $<0.001$ \\
\hline With & $3393(3.67)$ & $679(2.92)$ & $176(3.21)$ & $1625(3.64)$ & $913(4.77)$ & \\
\hline \multicolumn{7}{|c|}{ Acquired immune deficiency syndrome } \\
\hline Without & $92,407(99.94)$ & $23,229(99.96)$ & $5482(99.93)$ & $44,579(99.94)$ & $19,117(99.93)$ & 0.725 \\
\hline With & $53(0.06)$ & $10(0.04)$ & $4(0.07)$ & $25(0.06)$ & $14(0.07)$ & \\
\hline \multicolumn{7}{|c|}{ Respiratory dysfunction } \\
\hline Without & $82,866(89.62)$ & $20,958(90.18)$ & $4973(90.65)$ & $39,929(89.52)$ & $17,006(88.89)$ & $<0.001$ \\
\hline With & $9594(10.38)$ & $2281(9.82)$ & $513(9.35)$ & $4675(10.48)$ & $2125(11.11)$ & \\
\hline \multicolumn{7}{|l|}{ Sepsis } \\
\hline Without & $83,296(90.09)$ & $21,343(91.84)$ & $4,957(90.36)$ & $39,992(89.66)$ & $17,004(88.88)$ & $<0.001$ \\
\hline With & 9164 (9.91) & $1896(8.16)$ & $529(9.64)$ & $4612(10.34)$ & $2127(11.12)$ & \\
\hline \multicolumn{7}{|c|}{ Other diseases of lung (excluding respiratory dysfunction) } \\
\hline Without & $81,551(88.20)$ & $20,986(90.31)$ & $4997(91.09)$ & $39,582(88.74)$ & $15,986(83.56)$ & $<0.001$ \\
\hline With & $10,909(11.80)$ & $2253(9.69)$ & 489 (8.91) & $5022(11.26)$ & $3145(16.44)$ & \\
\hline
\end{tabular}

Note: P, chi-square and Fisher exact tests for categorical variables and one-way ANOVA with Scheffe's post hoc test for continuous variables.

patients who concomitantly used an Inhaled corticosteroid. ${ }^{9}$ Some studies have reported that statins lost their beneficial effects with increasing COPD severity or without cardiovascular comorbidity. ${ }^{2,3}$ The STATCOPE study focused on moderate to severe COPD and found that statins did not affect the exacerbation rate. ${ }^{12}$ Another two randomized controlled trials pointed out statins did not improve in pulmonary function. ${ }^{16,17}$ 


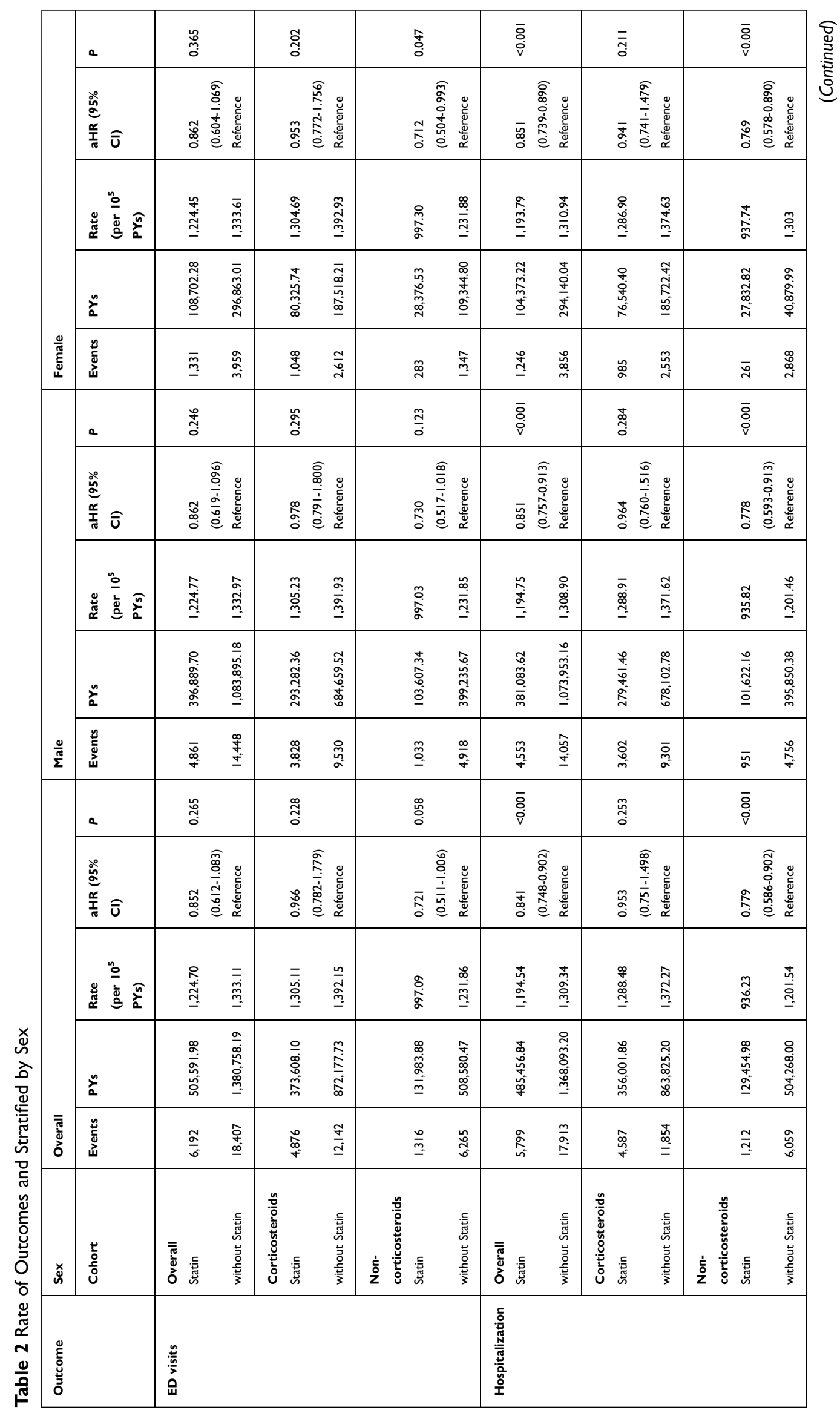




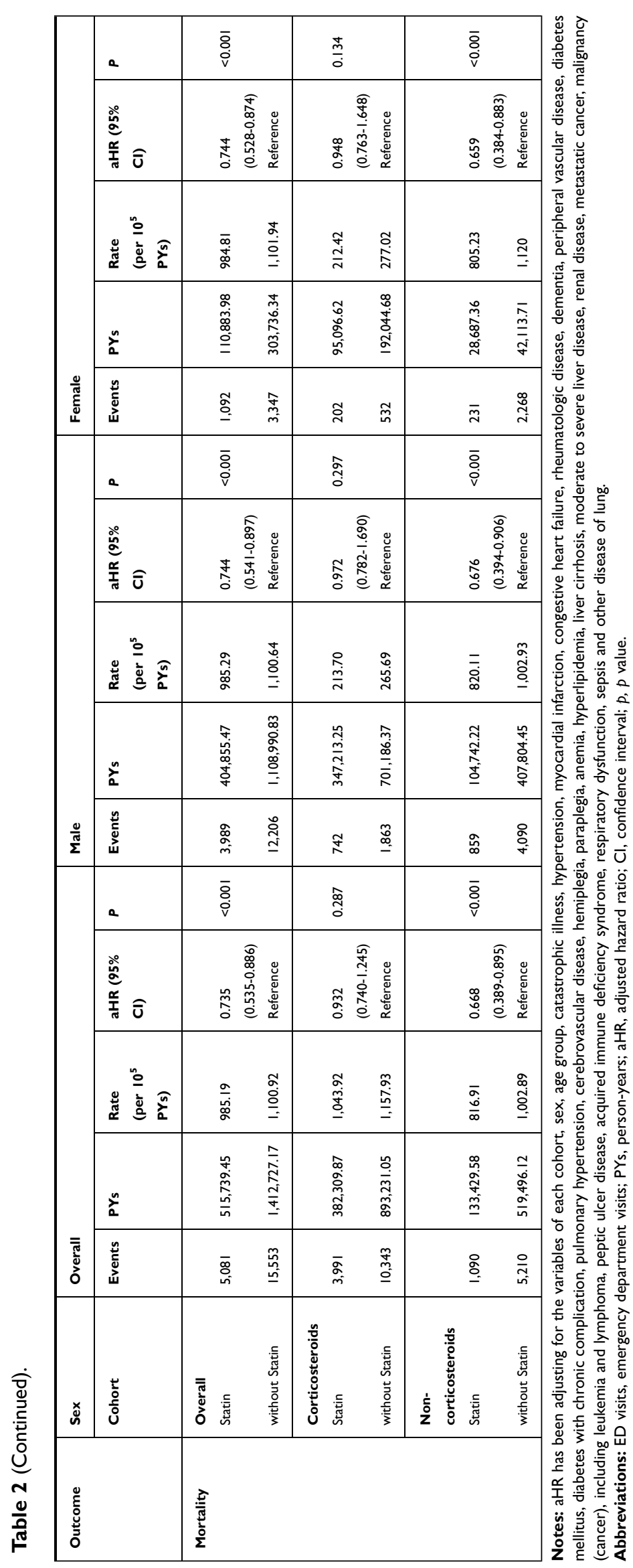




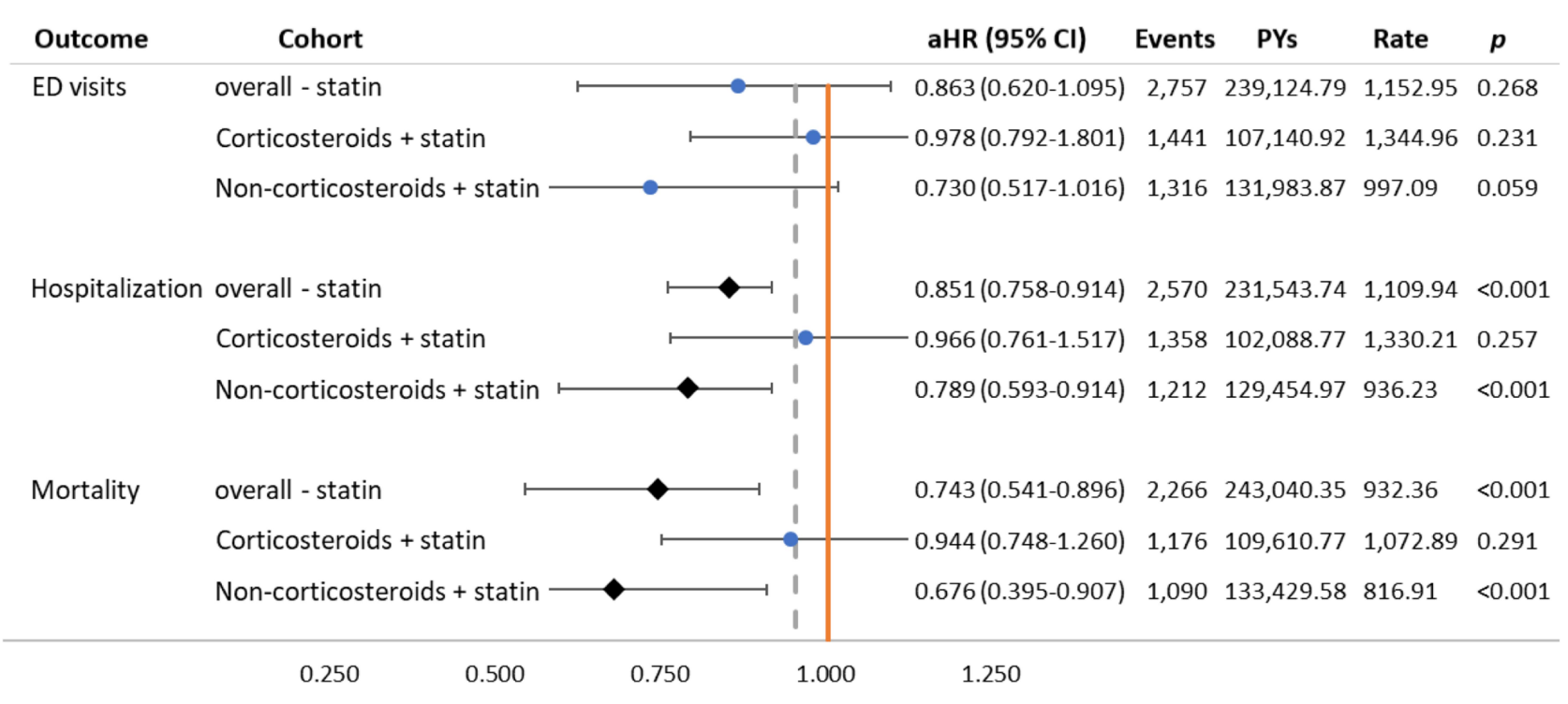

Figure 2 Forest plot of analysis by propensity score matching $\mathrm{I}: \mathrm{I}$.

Note: $\bullet$ means statistically significant $(p$ values $<0.05)$ and $\bullet$ means not.

Abbreviations: ED visits, emergency department visits; aHR, adjusted hazard ratio: adjusted for the variables listed in the Table S2; $\mathrm{Cl}$, confidence interval.

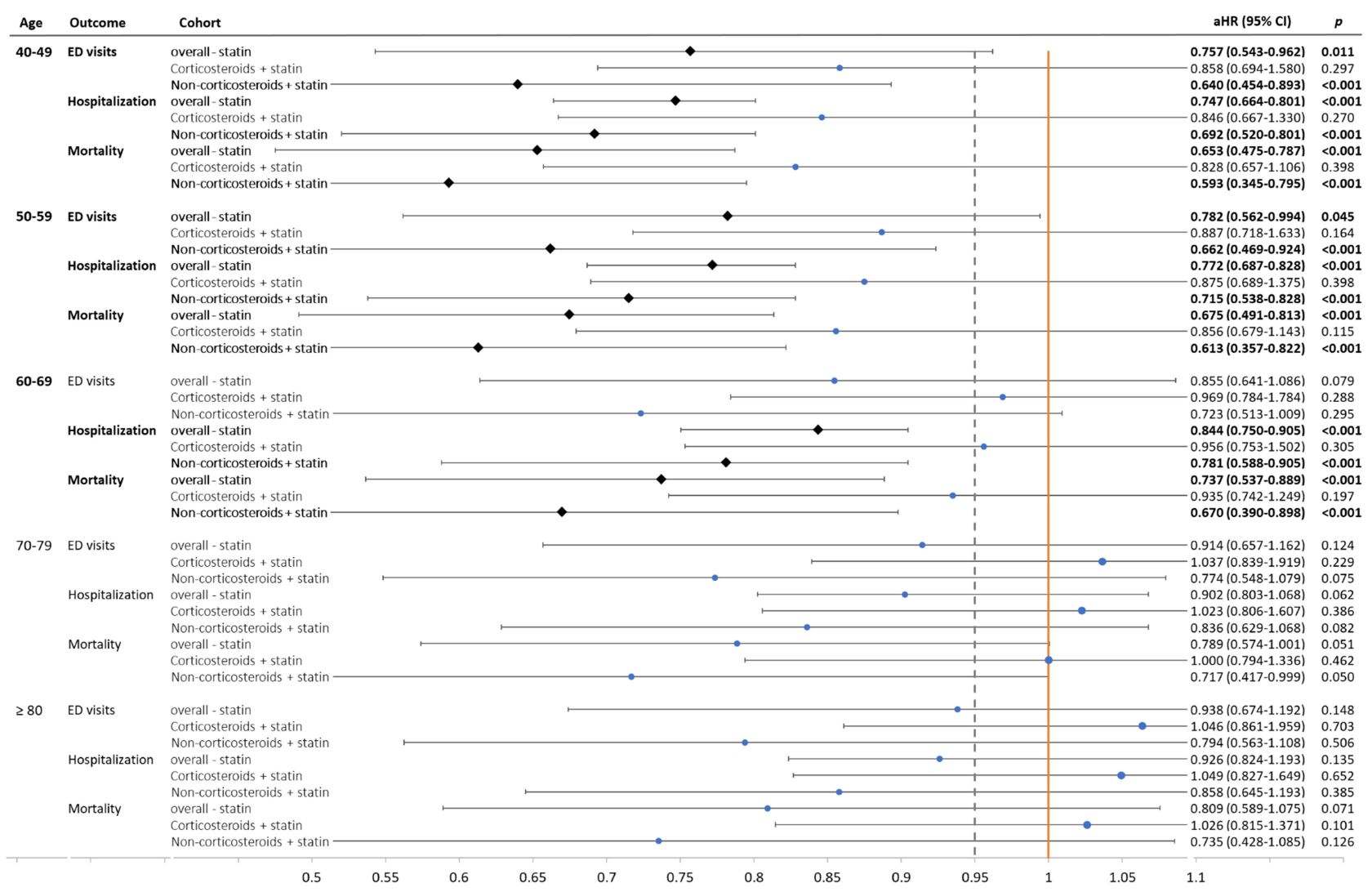

Figure 3 Forest plot of age-stratified.

Notes: $\bullet$ and boldface of the word mean statistically significant ( $\mathrm{p}$ values $<0.05)$; $\bullet$ means not statistically significant.

Abbreviations: ED visits, emergency department visits; aHR, adjusted hazard ratio: adjusted for the variables listed in the Table S2; $\mathrm{Cl}$, confidence interval. 
Some of the studies demonstrated that statins are particularly effective in COPD patients with cardiovascular comorbidity. ${ }^{2,9,18}$ There is no doubt that COPD and cardiovascular comorbidity are often co-existed, especially more severe severity of COPD. But the benefits of statins for patients with COPD and cardiovascular disease coexisted is from the treatment of COPD or treatment of cardiovascular disease have still not been clarified. ${ }^{19}$ Moreover, COPD exacerbations (respiratory symptoms) may be caused by exacerbation of comorbidities, not COPD itself. ${ }^{20}$ Those confused the beneficial effects of statins that are contributed by the management of COPD or the relative effect of comorbidities control. We adjusted dozens of comorbidities and factors listed in Table S2 to understand the beneficial effects of statins for patients with COPD in the real-world. This study reinforced most comorbidities aggravated the COPD exacerbation and mortality rate. These included not only cardio- or cerebrovascular disease but also liver or kidney function insufficiency, dementia, rheumatologic disease, other lung diseases, and even peptic ulcer disease. Overall, it seems that statins were more effective for COPD in observational studies $^{2-11}$ or a specific population (co-exited with cardiovascular comorbidity), ${ }^{9-11,13,14,18,19}$ but not in randomized controlled trials. ${ }^{12,16,17,21}$ Corticosteroids usage may be an important factor that affects the effectiveness of statins in COPD.

We found that statins gradually became less effective with patient age in COPD. The most effective group of statins was the youngest group in our study (40-49-yearold group), including ED visits, hospitalization rate, and mortality rate (Figure 3 and Supplementary Table S3). We also found that statins lost their beneficial effects in patients $>70$ years. These limitations will narrow the use of statins for COPD to less than half the overall population. If we consider the effect of age and exclude the patients who have already used corticosteroids, statins are only efficacious in $14 \%$ of COPD patients.

The pharmacological mechanism of statins is not yet completely understood. Presently, statins have been proven to provide multiple effects, not just hypolipidemic effects. Statins can be used for venous thromboembolism, ${ }^{22,23}$ cancers, ${ }^{24}$ stroke, ${ }^{25-27,31}$ and even as an antiarrhythmic drug. ${ }^{28-30}$ However, statins have already been proven to reduce stroke risk and atherosclerosis mainly by reducing low-density lipoprotein cholesterol, which is exactly the effect of hypolipidemic agents and not by reducing inflammation. ${ }^{31}$ In our study, we cannot determine which pharmacological mechanism was involved in the reduction of COPD exacerbation and mortality rates by statins. Whether this is a direct or indirect result remains a mystery.

A strength of our study that it is the first to show that statins lost beneficial effects in patients with COPD on corticosteroids. Moreover, statins provided similar effects on COPD male and female patients, but the beneficial effects gradually diminished with age. These findings will limit the applicability of statins to COPD. Furthermore, we identified COPD patients by three or more medical codes for COPD to validate the ICD-9-CM codes in Taiwan NHIRD and to exclude COPD patients before the index date. ${ }^{32}$ We used Cox regression models and adjusted numerous variable factors to investigate and clarify the effects of statins. We excluded any outcomes occurring $\leq 60$ days and rechecked the medication used by each patient for every outcome to ensure that the outcomes were related to statins and corticosteroids to avoid immortal time bias. These criteria should have made our study more rigorous and our results more reliable. Finally, the reproducibility of research results makes our conclusions more credible.

This study also had some limitations that should be considered when interpreting the results. First, we could not describe important clinical characteristics or links to laboratory data, such as inflammatory markers or pulmonary function test results, which limited our ability to identify causation and explain direct or indirect outcomes among our findings. However, this is a 16 -year cohort study that included all patients diagnosed with COPD in Taiwan and used data from a two million-person sample, the sample size is sufficient to make our results representative and applicable to a population of 23 million in Taiwan. Second, we did not further analyze the dose-dependent effects of statins or corticosteroids with each other, or stratified by topically or systemically corticosteroids used in COPD patients. Even so, sensitivity analyses showed that our findings were sufficiently robust to conclude. Finally, we could not assess the actual medicinal compliance rate. However, according to the policy of Taiwan's National Health Insurance Agency, patients must obtain "refillable prescriptions for patients with chronic illnesses" and revisit medical institutions more than three times per year. This requirement would lead to good compliance, and we assumed that most patients followed this policy. From this study, we confirmed that corticosteroids masked the beneficial effects of statins on COPD and corticosteroids may not be the only factor influencing the effects of statins. 


\section{Conclusion}

Statins decreased hospitalization and mortality rates in COPD patients not taking corticosteroids but were not efficacious in patients on corticosteroids therapy. Furthermore, the beneficial effects of statins gradually decreased with patient age. Based on the findings, statins used in COPD patients may need to consider the patient's age and corticosteroids used or not.

\section{Abbreviations}

COPD, chronic obstructive pulmonary disease; GOLD, Global Initiative for Chronic Obstructive Lung Disease; LHID, Longitudinal Health Insurance Database; NHIRD, National Health Insurance Research Database; ED, emergency department; aHR, adjusted hazard ratios; CI, confidence interval.

\section{Acknowledgments}

We appreciate the Health and Welfare Data Science Center, Ministry of Health and Welfare (HWDC, MOHW), Taiwan, for providing the National Health Insurance Research Database (NHIRD) and supported in part by the TriService General Hospital (TSGH-B-110012).

Kwua-Yun Wang and Wu-Chien Chien have equal contributions to this study.

\section{Disclosure}

The authors report no conflicts of interest in this work.

\section{References}

1. Vogelmeier CF, Criner GJ, Martinez FJ, et al. Global strategy for the diagnosis, management, and prevention of chronic obstructive lung disease 2017 report. GOLD executive summary. Am J Respir Crit Care Med. 2017;195(5):557-582. doi:10.1164/rccm.201701-0218PP

2. Ingebrigtsen TS, Marott JL, Nordestgaard BG, Lange P, Hallas J, Vestbo J. Statin use and exacerbations in individuals with chronic obstructive pulmonary disease. Thorax. 2015;70(1):33-40. doi:10.1136/thoraxjnl-2014-205795

3. Wang M-T, Lo Y-W, Tsai C-L, et al. Statin use and risk of COPD exacerbation requiring hospitalization. Am $\quad J$ Med. 2013;126 (7):598-606. doi:10.1016/j.amjmed.2013.01.036

4. Cao C, Wu Y, Xu Z, et al. The effect of statins on chronic obstructive pulmonary disease exacerbation and mortality: a systematic review and meta-analysis of observational research. Sci Rep. 2015;5:16461. doi:10.1038/srep16461

5. Young R, Hopkins R, Eaton T. Pharmacological actions of statins: potential utility in COPD. Eur Respir Rev. 2009;18(114):222-232. doi: $10.1183 / 09059180.00005309$

6. Janda S, Park K, FitzGerald JM, Etminan M, Swiston J. Statins in COPD: a systematic review. Chest. 2009;136(3):734-743. doi:10.1378/chest.09-0194

7. Dobler CC, Wong KK, Marks GB. Associations between statins and COPD: a systematic review. BMC Pulm Med. 2009;9(1):32. doi:10.1186/1471-2466-9-32
8. Lahousse L, Loth DW, Joos GF, et al. Statins, systemic inflammation and risk of death in COPD: the Rotterdam study. Pulm Pharmacol Ther. 2013;26(2):212-217. doi:10.1016/j.pupt.2012.10.008

9. Lin C-M, Yang T-M, Yang Y-H, et al. Statin use and the risk of subsequent hospitalized exacerbations in COPD patients with frequent exacerbations. Int $J$ Chron Obstruct Pulmon Dis. 2020;15:289. doi:10.2147/COPD.S229047

10. Young RP, Hopkins RJ, Agusti A. Statins as adjunct therapy in COPD: how do we cope after STATCOPE? Thorax. 2014;69:891-894. doi:10.1136/thoraxjnl-2014-205814

11. Kalchiem-Dekel O, Reed RM. Statins in COPD: life after STATCOPE. Chest. 2017;152(3):456-457. doi:10.1016/j. chest.2017.04.156

12. Criner GJ, Connett JE, Aaron SD, et al. Simvastatin for the prevention of exacerbations in moderate-to-severe COPD. $N$ Engl J Med. 2014;370(23):2201-2210. doi:10.1056/NEJMoa1403086

13. Søyseth V, Brekke P, Smith P, Omland T. Statin use is associated with reduced mortality in COPD. Eur Respir J. 2007;29(2):279-283. doi:10.1183/09031936.00106406

14. Bartziokas K, Papaioannou AI, Minas M, et al. Statins and outcome after hospitalization for COPD exacerbation: a prospective study. Pulm Pharmacol Ther. 2011;24(5):625-631. doi:10.1016/j.pupt.2011.06.003

15. Frost FJ, Petersen H, Tollestrup K, Skipper B. Influenza and COPD mortality protection as pleiotropic, dose-dependent effects of statins. Chest. 2007;131(4):1006-1012. doi:10.1378/chest.06-1997

16. Neukamm A, Høiseth A, Einvik G, et al. Rosuvastatin treatment in stable chronic obstructive pulmonary disease (RODEO): a randomized controlled trial. Intern Med J. 2015;278(1):59-67. doi:10.1111/joim.12337

17. Balaguer C, Peralta A, Ríos Á, et al. Effects of simvastatin in chronic obstructive pulmonary disease: results of a pilot, randomized, placebo-controlled clinical trial. Contemp Clin Trials Commun. 2016;2:91-96. doi:10.1016/j.conctc.2015.12.008

18. Zhang W, Zhang Y, Li C-W, Jones P, Wang C, Fan Y. Effect of statins on COPD: a meta-analysis of randomized controlled trials. Chest. 2017;152(6):1159-1168. doi:10.1016/j.chest.2017.08.015

19. André S, Conde B, Fragoso E, Boléo-Tomé J, Areias V, Cardoso J. COPD and cardiovascular disease. Pulmonology. 2019;25 (3):168-176. doi:10.1016/j.pulmoe.2018.09.006

20. Beghé B, Verduri A, Roca M, Fabbri LM. Exacerbation of respiratory symptoms in COPD patients may not be exacerbations of COPD. Eur Respir J. 2013;41(4):993-995. doi:10.1183/09031936.00180812

21. So JY, Dhungana S, Beros JJ, Criner GJ. Statins in the treatment of COPD and asthma-where do we stand? Curr Opin Pharmacol. 2018;40:26-33. doi:10.1016/j.coph.2018.01.001

22. Schmidt M, Cannegieter SC, Johannesdottir SA, Dekkers OM, HorváthPuhó E, Sørensen HT. Statin use and venous thromboembolism recurrence: a combined nationwide cohort and nested case-control study. $J$ Thromb Haemost. 2014;12(8):1207-1215. doi:10.1111/jth. 12604

23. Wallace A, Albadawi H, Hoang P, et al. Statins as a preventative therapy for venous thromboembolism. Cardiovasc Diagn Ther. 2017;7(Suppl 3):S207. doi:10.21037/cdt.2017.09.12

24. Zhong S, Zhang X, Chen L, Ma T, Tang J, Zhao J. Statin use and mortality in cancer patients: systematic review and meta-analysis of observational studies. Cancer Treat Rev. 2015;41(6):554-567. doi:10.1016/j.ctrv.2015.04.005

25. Hong K-S, Lee JS. Statins in acute ischemic stroke: a systematic review. J Stroke. 2015;17(3):282. doi:10.5853/jos.2015.17.3.282

26. Lee M, Saver JL, Wu YL, et al. Utilization of statins beyond the initial period after stroke and 1-year risk of recurrent stroke. $J \mathrm{Am}$ Heart Assoc. 2017;6(8):e005658. doi:10.1161/JAHA.117.005658

27. Valentino M, Al Danaf J, Panakos A, Ragupathi L, Duffy D, Whellan D. Impact of the 2013 American College of Cardiology/American Heart Association cholesterol guidelines on the prescription of high-intensity statins in patients hospitalized for acute coronary syndrome or stroke. Am Heart J. 2016;181:130-136. doi:10.1016/j.ahj.2016.07.024 
28. Rezaei Y, Gholami-Fesharaki M, Dehghani MR, Arya A, Haghjoo M, Arjmand N. Statin antiarrhythmic effect on atrial fibrillation in statin-naive patients undergoing cardiac surgery: a meta-analysis of randomized controlled trials. J Cardiovasc Pharmacol Ther. 2016;21 (2):167-176. doi:10.1177/1074248415602557

29. Verrier RL. Statins protect against arrhythmogenic calcium alternans in the post-myocardial infarction diabetic heart: pleiotropy on steroids. Heart Rhythm. 2017;14(9):1417-1418. doi:10.1016/j. hrthm.2017.06.010

30. Kourliouros A, De Souza A, Roberts N, et al. Dose-related effect of statins on atrial fibrillation after cardiac surgery. Ann Cardiothorac Surg. 2008;85(5):1515-1520.
31. Amarenco P, Labreuche J, Lavallée P, Touboul P-J. Statins in stroke prevention and carotid atherosclerosis: systematic review and up-todate meta-analysis. Stroke. 2004;35(12):2902-2909. doi:10.1161/01. STR.0000147965.52712.fa

32. Ho T-W, Ruan S-Y, Huang C-T, Tsai Y-J, Lai F, Yu C-J. Validity of ICD9-CM codes to diagnose chronic obstructive pulmonary disease from National Health Insurance claim data in Taiwan. Int J Chron Obstruct Pulmon Dis. 2018;13:3055. doi:10.2147/COPD.S174265

\section{Publish your work in this journal}

The International Journal of COPD is an international, peer-reviewed journal of therapeutics and pharmacology focusing on concise rapid reporting of clinical studies and reviews in COPD. Special focus is given to the pathophysiological processes underlying the disease, intervention programs, patient focused education, and self management protocols. This journal is indexed on PubMed Central, MedLine and CAS. The manuscript management system is completely online and includes a very quick and fair peer-review system, which is all easy to use. Visit http://www.dovepress.com/testimonials.php to read real quotes from published authors. 